\title{
On-Line Single Droplet Deposition for MALDI Mass Spectrometry
}

\author{
Xia Zhang, Damien A. Narcisse, and Kermit K. Murray \\ Department of Chemistry, Louisiana State University, Baton Rouge, Louisiana, USA
}

\begin{abstract}
A single droplet generator was coupled with a rotating ball inlet matrix-assisted laser desorption/ionization (MALDI) time of flight (TOF) mass spectrometer. Single droplets with 100 picoliter volume were ejected by a piezoelectric-actuated droplet generator and deposited onto a matrix-coated rotating stainless steel ball at atmospheric pressure. The single droplet deposit was transported to the vacuum side of the instrument where ionization was accomplished using a UV pulsed laser. Using this on-line interface, it was possible to obtain protonated molecule signal from as little as $10 \mathrm{fmol}$ analyte. (J Am Soc Mass Spectrom 2004, 15, 1471-1477) (C 2004 American Society for Mass Spectrometry
\end{abstract}

$\mathrm{M}$ atrix-assisted laser desorption/ionization (MALDI) mass spectrometery is currently employed in many areas of biomolecular analysis because of high sensitivity, low sample consumption, and high tolerance to impurities [1]. A typical MALDI sample preparation involves the deposition of microliter sample volumes on a metal target to produce a spot several millimeters in diameter. Because the focused spot size ionization laser is typically a few tenths of a millimeter in diameter, it is possible to improve the sensitivity of a MALDI analysis by reducing the size of the sample deposit. One way to minimize spot size is patterned hydrophobic surfaces. MALDI sample targets coated with a thin hydrophobic layer and an array of $200 \mu \mathrm{m}$ uncoated gold spots have recently been demonstrated [2]. The water-repellent layer, in combination with the hydrophilic nature of the bare metal spot, minimizes the final sample spot size and thus the quantity of sample required for analysis.

Further improvements in sensitivity as well as speed of analysis can be achieved through single droplet production and deposition [3]. A number of recent studies have demonstrated the utility of depositing small and uniform droplets on sample targets for MALDI analysis [4-6]. Small droplets can be generated by thermal, piezoelectric, electrostatic, or acoustic methods, in which single droplets are ejected by a brief pressure pulse [7]. The most widely used mechanism for droplet production in MALDI applications has been piezoelectric particle generation $[4,6,8-10]$. In these devices, a radially contracting piezoelectric cylinder or flexing diaphragm causes a rapid pressure pulse that releases a single droplet through an orifice upon the

Published online September 11, 2004

Address reprint requests to Professor K. K. Murray, Department of Chemistry, Louisiana State University, 232 Choppin Hall, Baton Rouge, LA 70803, USA. E-mail: kkmurray@lsu.edu application of a voltage pulse. Because the liquid droplet has a sample volume between tens and hundreds of picoliters, the resulting spot diameter, typically ranging from 100 to $500 \mu \mathrm{m}$ can be created on the target. The quantity of analyte in a given spot can be varied by depositing multiple droplets on the same target spot.

Several methods that are departures from the piezoelectric deposition approach have recently been reported. A novel liquid deposition system for off-line LC/MALDI-MS employs a pulsed electric field to transfer the eluents of multiple chromatographic columns as a series of droplets directly onto a MALDI target plate without the column outlet touching the target surface [11]. A negative voltage pulse applied to the target stage causes charging of a suspended droplet, which is then pulled to the target by the electric field. In another approach, an acoustic droplet generator uses a pressure pulse focused at the meniscus of the fluid to eject particles upward from the surface of the liquid [12]. Samples have also been deposited to MALDI targets using a heated capillary nebulizer that provides for rapid desolvation of effluent [13] and with a heated droplet interface that results in partial evaporation of the solvents from the hanging droplets and on the target plate, forming small analyte spots [14].

The ability to manipulate small sample volumes in the form of droplets affords unique opportunities for sample treatment on a nanoliter scale. In one example, nanoliter chemistry and droplet deposition were demonstrated for protein digestion and peptide mass mapping using the lysates of single mammalian cells [15]. Low-volume extraction for the analysis of hydrophobic peptides and phospholipids has also been demonstrated [16]. Single droplet deposition can be used for off-line coupling MALDI to liquid separations $[17,18]$ and reactors [19]. For example, an integrated microanalytical platform, which can analyze more than 25 protein samples per hour, has been developed for 
automated identification of proteins [19]. In this system, a microchip immobilized enzyme reactor for enzymatic digestion of protein samples was used with a piezoelectric microdispenser for interfacing to a MALDI-TOF MS. A piezoelectric flow-through microdispenser for interfacing capillary liquid chromatography (LC) with MALDI-TOF MS was recently demonstrated for the identification of biomolecules [20].

The above applications use single droplet deposition on a MALDI target with later off-line analysis by MALDI. However, single droplet deposition also has the potential for on-line analysis, in which the deposition and interrogation by mass spectrometry occur sequentially in real-time. Recently, a rotating quartz wheel on-line MALDI interface was reported, in which the liquid is deposited using a narrow fused silica capillary in contact with the wheel [21]. The system is compatible with crystalline matrixes because clogging at the capillary exit is prevented through the physical contact with the rotating wheel. A disadvantage of the system is the limited operation time: the wheel must be cleaned after it has made a $360^{\circ}$ cycle in about $3 \mathrm{~min}$. In a related work, a rotating ball inlet interface, capable of continuously introducing solutions directly into a TOF MS system, was demonstrated for on-line MALDI [22]. This interface is compatible with any crystalline MALDI matrix and doesn't suffer from sample memory effects. The major drawbacks of this system are the relatively high dead volumes and the requirement for direct contact between the analyte solution and the ball.

We have developed a new version of the rotating ball inlet that addresses the design issues of the previous version [23]. The system is constructed so that more than half of the ball interface is exposed to atmospheric pressure and can be used for either direct contact or non-contact sample deposition. In this article, we describe a single-droplet non-contact deposition method for the rotating ball inlet. Matrix spots were formed on the ball using a $50 \mu \mathrm{m}$ capillary. Individual picoliter volume droplets of analyte were deposited on top of the matrix spots using a piezoelectric single droplet generator. The sample deposit was rotated into the vacuum side of the instrument and ions were formed by UV MALDI and analyzed by TOF MS. Results comparing on-line and off-line single droplet deposition MALDI analysis are described below.

\section{Experimental}

The rotating ball inlet TOF mass spectrometer is a modification of the instrument that was described previously $[22,23]$. The mass spectrometer is a $1 \mathrm{~m}$ linear time-of-flight (TOF) instrument with a specially constructed rotating ball interface, shown in Figure 1. The interface consists of a modified ISO-100 stainless steel flange mounted on an 8-inch conflat six-way cross. The flange is electrically isolated using a $25 \mathrm{~mm}$ thick Delrin (acetyl resin) insulator flange and is held at high voltage during operation. The flange is machined to accept the

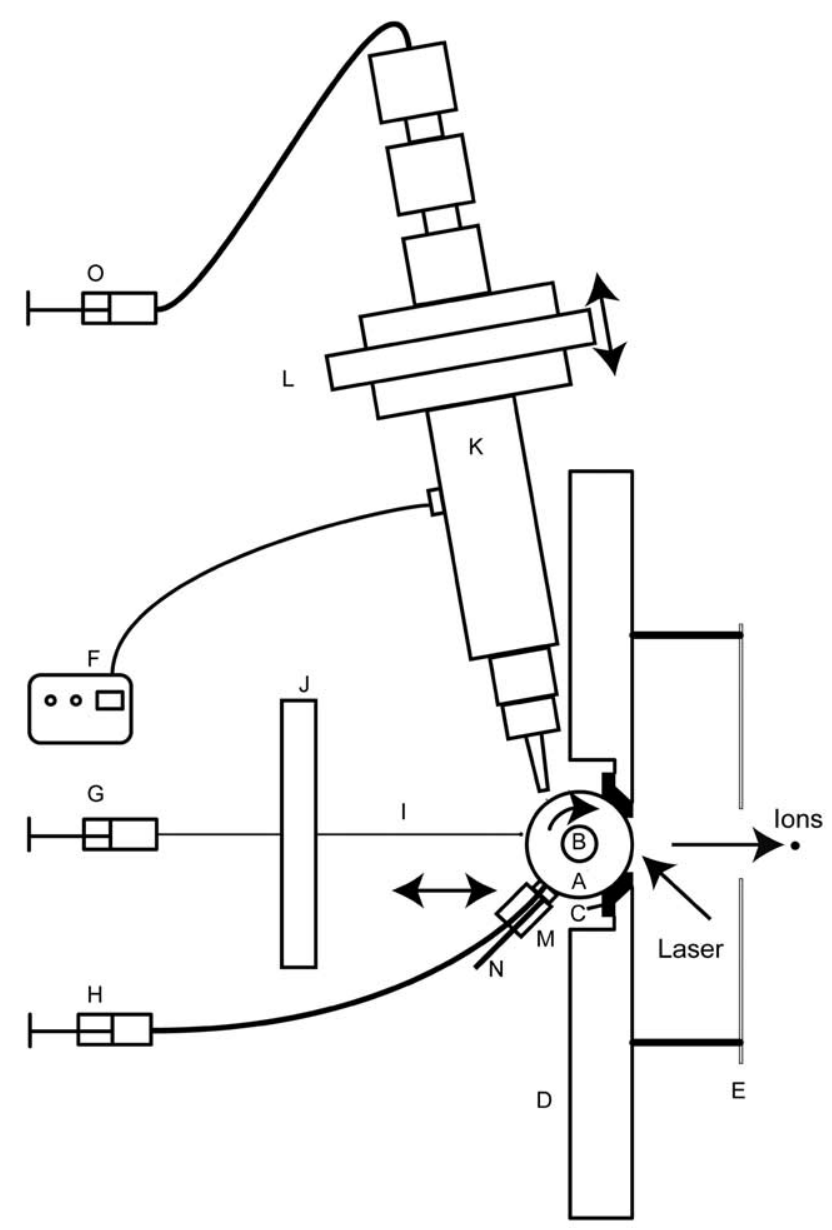

Figure 1. Schematic of on-line single droplet deposition device: (a) $19 \mathrm{~mm}$ diameter stainless steel ball; (b) drive shaft; (c) gasket; (d) ISO-100 flange; (e) ground grid; (f) droplet generation electronics; (g) syringe pump for matrix solution; (h) syringe pump for solvent; (i) capillary; (j) translation stage for capillary; (k) droplet generator; (1) translation stage for droplet generator; $(\mathbf{m})$ cleaning system (Teflon holder and felt); (n) PEEK plastic tube for waste; (o) syringe pump for analyte solution.

rotating ball, which allows samples to be introduced on the atmospheric pressure side of the flange. A type 316 stainless steel ball 0.75 inch in diameter (Small Parts, Miami Lakes, FL) was press fit with a 0.25 inch shaft and attached to a multi-speed transmission gearbox scavenged from a syringe pump (Model 901, Harvard Apparatus, Holliston, MA). The ball and the shaft are clamped onto the interface flange by two stainless steel holders with sintered bronze bearings. The ion source chamber is constructed so that the rotating ball forms a vacuum seal on the interface flange with a Teflon gasket between the ball and flange. The ion source chamber is evacuated with a $1500 \mathrm{~L} / \mathrm{s}$ diffusion pump and maintains a pressure lower than $5 \times 10^{-6}$ torr during operation.

For droplet deposition, the rotation rate of the ball was $0.12 \mathrm{rpm}$. Prior to droplet deposition, a matrix spot was deposited on the ball using a $50 \mu \mathrm{m}$ i.d. fused silica capillary held horizontally using an $\mathrm{x}-\mathrm{y}-\mathrm{z}$ translation 
stage (Model LP-1, Newport, Irvine, CA). The analyte was delivered from a $100 \mu \mathrm{M}$ solution at a flow rate of $1.5 \mu \mathrm{L} / \mathrm{min}$ using a syringe pump (Model 55-2222; Harvard Apparatus). Matrix spots were created by intermittently contacting the ball with the matrix capillary. Sample droplets were ejected onto the matrix spots as they rotated below the droplet generator (model 201, Uni-photon Systems, Brooklyn, NY), which was held in a second $x-y-z$ translation stage. The droplet generator was pointed downward toward the ball at a $10^{\circ}$ angle from vertical and immediately adjacent to the flange. The distance between the ball and droplet generator tip was $2.5 \mathrm{~mm}$. The analyte solution was introduced into the droplet generator with a syringe pump (Model 55-2275, Harvard Apparatus). Visualization of sample droplet was obtained using an external video camera and macro zoom lens.

Samples deposited on the ball were rotated into the vacuum of the mass spectrometer where they were ionized by a $10 \mathrm{~Hz}$ pulsed $355 \mathrm{~nm}$ Nd:YAG laser (Continuum Minilite, Santa Clara, CA). The laser was focused to a spot size of $100 \mu \mathrm{m} \times 300 \mu \mathrm{m}$, as determined using laser burn paper and a measuring magnifier. A video camera and macroscopic lens were used to monitor the laser alignment on the sample. A solvent-saturated felt pad was held against the surface of the ball on the atmospheric side to clean the surface after it passed out of the ionization chamber.

Ions were accelerated into the mass spectrometer by a 16 to $20 \mathrm{kV}$ single-stage extraction and detected with an $18 \mathrm{~mm}$ bipolar time of flight detector (Burle, Sturbridge, MA). Data were recorded with a $500 \mathrm{MHz}$ digital oscilloscope (LT372; LeCroy, Chestnut Ridge, NY). Mass spectra were averaged on the oscilloscope and transferred to a computer using a general-purpose interface bus (GPIB) connection controlled by in-house software (Lab View, National Instruments, Austin, TX). All mass spectra shown below resulted from an average of five laser shots.

For comparison with the on-line rotating ball results, single droplet deposits were interrogated off-line using a commercial MALDI TOF time-of flight mass spectrometer (Omniflex, Bruker, Billerica, MA). The off-line instrument is equipped with a $337 \mathrm{~nm}$ nitrogen laser and can be operated in both linear and reflectron modes. For the off-line results shown below, the laser was operated at a repetition rate of $2 \mathrm{~Hz}$ and the instrument was operated in linear mode. The mass spectra were a sum of 20 laser shots. For off-line deposition, the matrix solution was placed on the target by depositing $0.27 \mu \mathrm{L}$ of a $50 \mathrm{mM}$ matrix solution using a $50 \mu \mathrm{m}$ i.d. capillary and allowing it to air dry. Following this, a single droplet of analyte (at $100 \mu \mathrm{M}$ concentration) was deposited onto the matrix-coated sample target. The distance between the glass tip and MALDI target was $2.5 \mathrm{~mm}$.

Single droplet sample preparations were collected on silicon wafers and analyzed by scanning electron microscopy (SEM). Prior to SEM, the samples were de- gassed and sputter coated with a $20 \mathrm{~nm}$ gold/palladium $(60 / 40)$ layer using a sputter coater (S-150B; Edwards, Crawley, UK). The samples were mounted on aluminum supports with conductive tape and examined at $15 \mathrm{kV}$ with a scanning electron microscope (S-260; Cambridge Instruments, Cambridge, UK) located in the LSU Socolofsky Microscopy Center.

The analytes angiotensin I (A-9650, Sigma, St. Louis, MO), bradykinin (B-3259, Sigma), substance P (S-6883, Sigma), bovine insulin (I-5500, Sigma), cytochrome $c$ (C-2506, Sigma), and horse myoglobin (M-1882, Sigma) were used as obtained from the manufacturer. The matrixes used in this work were $\alpha$-cyano-4-hydroxycinnamic acid (CHCA, Sigma), 3,5-dimethoxy-4-hydroxy cinnamic acid (sinapic acid, Fluka, Ronkonkoma, NY), 2-(4-hydroxyphenylazo)-benzonic acid (HABA, Aldrich, Milwaukee, WI), 2,5-dihydroxy benzoic acid (DHB, Aldrich) and 3,4-dihydroxy-cinnamic acid (caffeic acid, Aldrich). The matrix solutions were prepared in methanol (200 Proof, AAPER Alcohol, Shelbyville, KY) with $1 \%$ trifluoroacetic acid (TFA, J. T. Baker, Pittsburgh, NJ) at concentrations of $50 \mathrm{mM}$. The analyte solutions were made by dissolving the analyte in distilled water (house supply) with $1 \%$ TFA at a concentration of $100 \mu \mathrm{M}$. A solution of $10 \%$ glycerol $(99.7 \%$, Fisher, Fairlawn, NJ) in distilled water was prepared to measure the droplet size.

\section{Results and Discussion}

Droplet size and deposition precision experiments were performed using a solution of $10 \%$ glycerol in water. This mixture was necessary to limit the evaporation rate of the solution at atmospheric pressure and room temperature during the measurements. The droplets were collected on a hydrophobic Parafilm surface (Pechiney Plastic Packaging, Chicago, IL), which resulted in nearly spherical droplets. The distance between the droplet generator glass tip and the surface was $2.5 \mathrm{~mm}$, the drive voltage was $70 \mathrm{~V}$, and the flow rate was $800 \mathrm{~nL} / \mathrm{min}$. In order to measure the droplet size, a series of small concentric circles were printed on white paper using a printer and placed under the Parafilm to be used as an internal ruler in the experiments. Sizes were determined both for single ejected droplets as well as for twenty droplets ejected onto a single spot and coalesced into a larger droplet. From these experiments it was found that, using the former method, the diameter of the droplets was $57 \pm 6 \mu \mathrm{m}(15$ measurements). This corresponds to a volume of $97 \pm 3$ $\mathrm{pL}$. With the latter method, the diameter of the droplet is $53 \pm 2 \mu \mathrm{m}$ (10 measurements) and the corresponding volume is $78 \pm 9 \mathrm{pL}$.

The droplet placement accuracy was measured by depositing 100 single droplets onto the printer paper without parafilm at a deposition rate of $1 \mathrm{~Hz}$. Here, the solution was $100 \mu \mathrm{M}$ angiotensin I in distilled water with $1 \%$ TFA at $1.5 \mu \mathrm{L} / \mathrm{min}$ and the droplet generator voltage was $80 \mathrm{~V}$. The droplet generator was sur- 


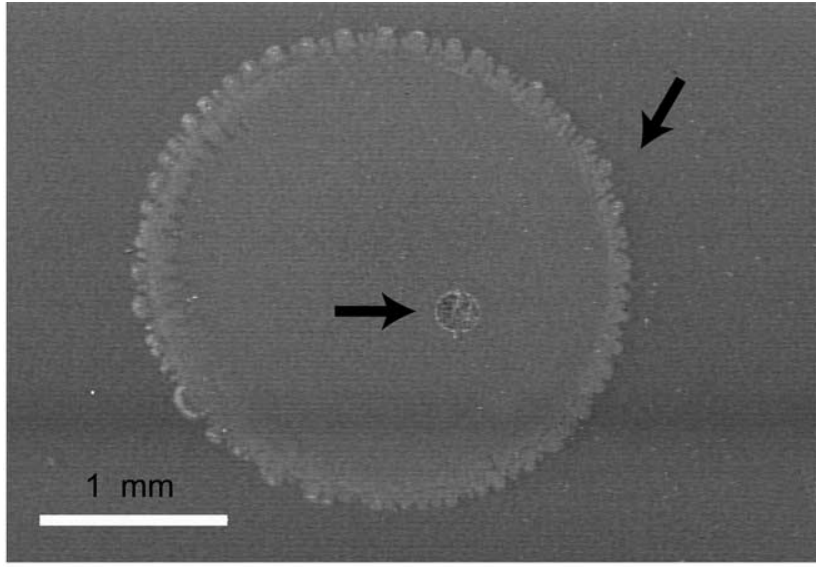

(a)

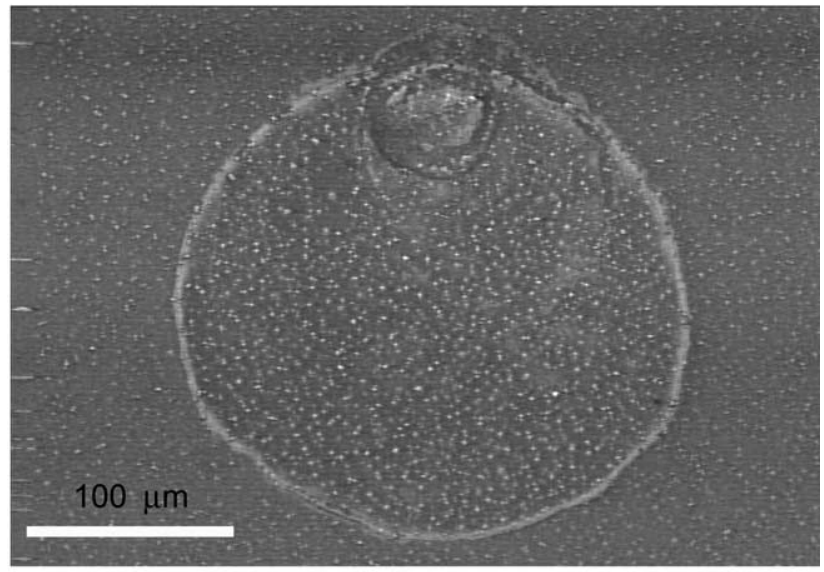

(b)

Figure 2. Scanning electron micrographs of matrix and analyte deposits showing (a) the matrix spot (analyte spot depicted with arrow at center) and (b) the analyte spot at a higher magnification.

rounded by vertical shields to minimize the effects of air currents in the laboratory. It was found that $94 \% \pm$ $2 \%$ (15 sets of measurements) of the droplets produced fell within a circular spot $400 \mu \mathrm{m}$ in diameter.

Scanning electron microscopy was used to measure the size of samples deposited on flat targets. Figure 2 contains scanning electron micrographs of matrix and analyte sample spots deposited on a silicon wafer under conditions similar to those used to obtain mass spectra (both off-line and on-line). Figure 2a shows the matrix spot after solvent evaporation, which was obtained by depositing $\sim 0.27 \mu \mathrm{L}$ from a $50 \mathrm{mM}$ matrix solution onto the silicon surface using $50 \mu \mathrm{m}$ i.d. capillary. The size of the matrix spot is approximately $2.5 \mathrm{~mm}$ in diameter. After the matrix spot had dried, an $80 \mathrm{pL}$ droplet of a $100 \mu \mathrm{M}$ angiotensin I solution (8 fmol) was deposited on the target. The image in Figure $2 b$ shows the analyte spot at higher magnification. The deposit is uniform with the exception of a single area on the circumference of the droplet that is believed to result from uneven solvent evaporation. The size of dried analyte spots obtained in this way was between 200 and $250 \mu \mathrm{m}$ diameter.

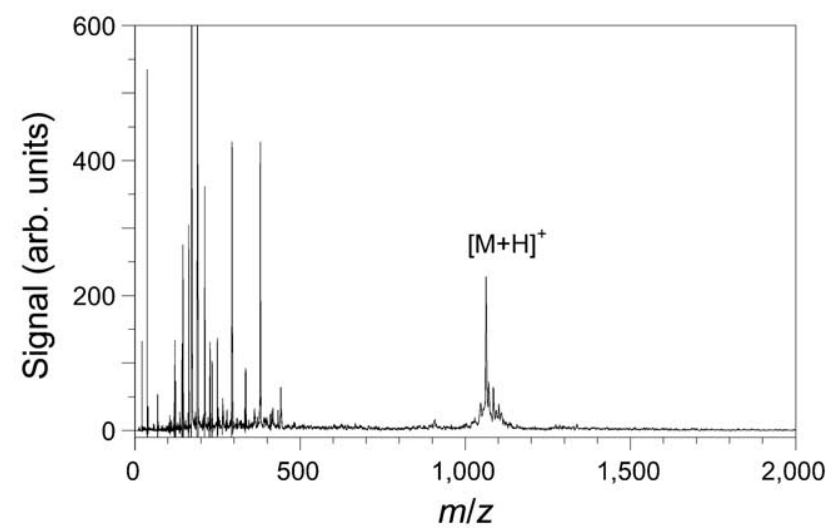

Figure 3. Mass spectrum obtained from a single droplet deposition of 8 fmol bradykinin on a MALDI target precoated with CHCA matrix.

Off-line droplet deposition onto MALDI targets was carried out on the Bruker Omniflex mass spectrometer. Figure 3 shows a mass spectrum obtained from a single droplet deposit of the peptide bradykinin $\left(M_{r}=1060.2\right)$, which was obtained using a $100 \mu \mathrm{M}$ solution to deposit 8 fmol analyte onto a target pre-coated with CHCA matrix. The most intense peak in the mass spectrum is protonated bradykinin $[\mathrm{M}+\mathrm{H}]^{+}$. The mass resolution of protonated bradykinin is about $2000(\mathrm{~m} / \Delta \mathrm{m}$, fwhm). In addition, peaks associated with sodium $[\mathrm{M}+\mathrm{Na}]^{+}$ and potassium $\left[\mathrm{M}+\mathrm{K}^{+}\right.$cation adducts are also observed. Peaks in the low-mass region correspond to sodium and potassium cations, matrix and matrix fragment ions. When using the $1 \mu \mathrm{L}$ dried droplet deposit of bradykinin, the detection limit for this instrument is approximately $50 \mathrm{fmol}$.

Several different standard MALDI matrixes, including sinapinic acid, DHB, HABA, and CHCA, were tested for the ionization of larger biomolecules. The CHCA matrix gave the best performance both in terms of ion signal as well as mass resolution. A mass spectrum obtained from a single droplet deposit of $8 \mathrm{fmol}$ bovine insulin $\left(\mathrm{M}_{\mathrm{r}}=5733.5\right)$ is shown in Figure $4 \mathrm{a}$. The $[\mathrm{M}+\mathrm{H}]^{+}$peak has the highest signal above $m / z=1000$ at a mass resolution of $1500 \mathrm{fwhm}$.

Higher mass proteins such as cytochrome $c\left(\mathrm{M}_{\mathrm{r}}=\right.$ $12,384)$ and myoglobin $\left(M_{r}=16,964\right)$ were also analyzed by the single droplet deposition method. Here, the analyte cytochrome $c$ was dissolved in a 2:1 ( $\mathrm{vol} /$ vol) mixture of $1.4 \%$ water aqueous trifluoroacetic acid and, acetonitrile and at a concentration of $100 \mu \mathrm{M}$. The mass spectrum of 8 fmol cytochrome $c$ from single droplet deposition on a MALDI target precoated with CHCA matrix is shown in Figure $4 \mathrm{~b}$. In this mass spectrum, the peak labeled $[\mathrm{M}+\mathrm{H}]^{+}$corresponds to protonated cytochrome $c$ and the peak labeled $[2 \mathrm{M}+$ $\mathrm{H}^{2+}$ corresponds to doubly protonated cytochrome $c$. The mass resolution is about $50(\mathrm{~m} / \Delta \mathrm{m}$, fwhm); the other matrixes gave a resolution of about 30 .

For on-line analysis, single droplets were deposited onto the rotating ball interface at atmospheric pressure 


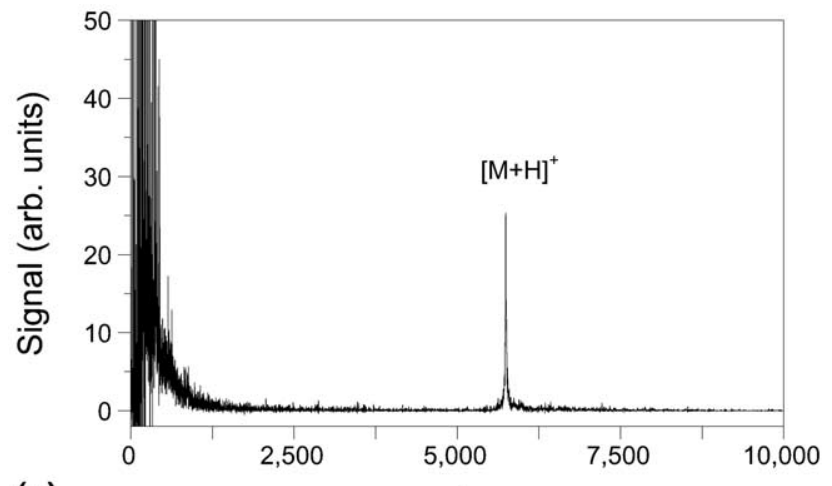

(a)

$\mathrm{m} / \mathrm{z}$

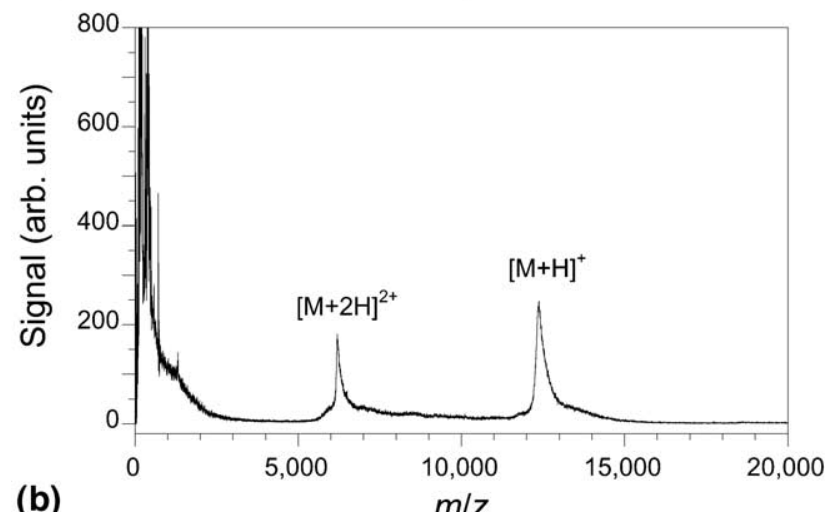

(b)

$m / z$

Figure 4. Off-Line MALDI mass spectra from a single droplet deposition of (a) bovine insulin and (b) cytochrome $c$.

and then rotated into the vacuum side for desorption and ionization. Before the deposition, the tip of droplet generator and the capillary was aligned to the center line of the stainless steel ball by adjusting the translation stages so that the sample spot could be irradiated without changing the laser alignment. The matrix solution was deposited onto the ball by intermittently moving the translation stage-mounted capillary. Figure 5 a depicts the mass spectrum of angiotensin I obtained in this manner. The spectrum is characterized by large protonated molecule peaks and less intense alkali metal adduct peaks. The mass resolution is 400 .

A mass spectrum of a single droplet containing 8 fmol of bovine insulin deposited on the ball coated with CHCA is shown in Figure $5 \mathrm{~b}$. The protonated insulin peak $[\mathrm{M}+\mathrm{H}]^{+}$and the doubly protonated $[\mathrm{M}+2 \mathrm{H}]^{2+}$ are observed. The mass resolution of protonated bovine insulin is 40 , lower than that obtained in the off-line single droplet deposition spectrum (Figure 4a). Figure $5 c$ contains a mass spectrum obtained from an $8 \mathrm{fmol}$ single droplet deposit of cytochrome $c$ on the rotating ball interface coated with CHCA. The doubly protonated peak $[\mathrm{M}+2 \mathrm{H}]^{2+}$ and singly protonated $[\mathrm{M}+\mathrm{H}]^{+}$ cytochrome $c$ peaks were observed at a mass resolution of 40. The lower resolution for the ball deposition (Figure 5) as compared to the off-line single droplet deposit (Figures 3 and 4) may result from field inhomogenieties in the ion extraction region [22]. Desorp-

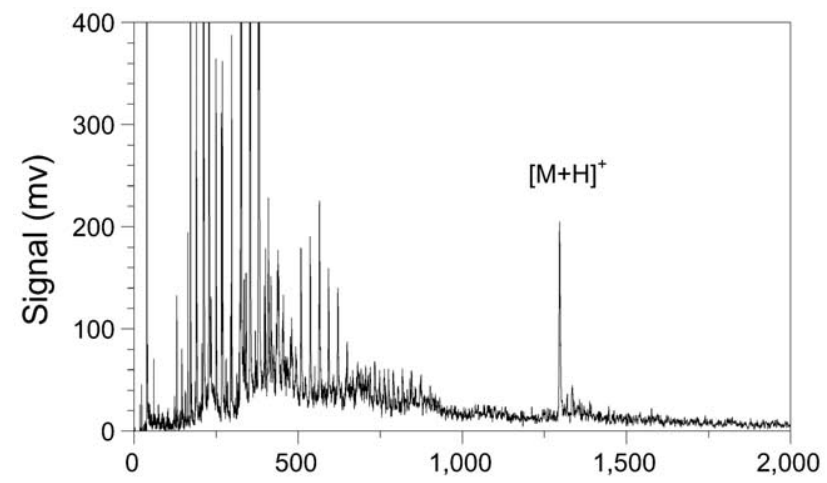

(a)

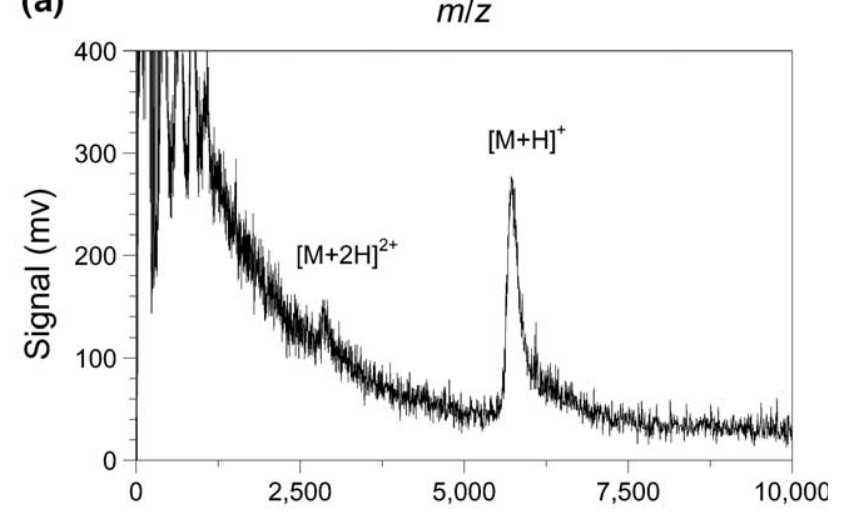

(b)

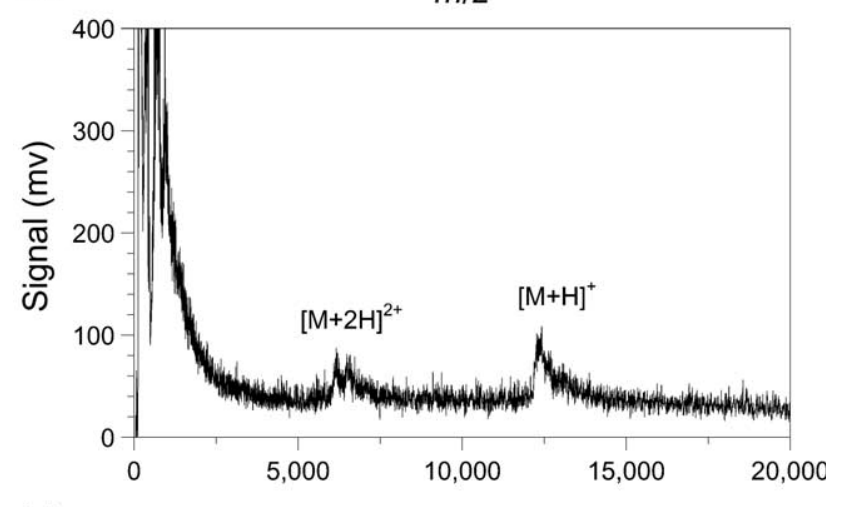

(c)

$m / z$

Figure 5. On-Line MALDI mass spectra from single droplet deposition on the rotating ball interface (a) angiotensin I, (b) bovine insulin, and (c) cytochrome $c$.

tion from the round surface of the ball may have an adverse effect on the spatial focusing of the ions and result in a broad distribution ion flight times in the accelerated packet. Furthermore, the rotating ball instrument does not utilize delayed ion extraction, which is used in the off-line instrument to improve the mass resolution [24]. Another possible reason for the lower mass resolution and signal is that when the sample spot passes through the gasket, the mechanical force between the gasket and the ball changes the surface morphology or adds contaminants to the deposit.

Mass resolution may be improved with delayed ion extraction, multiple stages of ion acceleration, or a 
reflectron flight tube. The curvature of the ball surface may ultimately limit the obtainable mass resolution, although this may be mitigated through electrostatic lensing and by limiting the exposed portion of the ball surface. Ultimately, the ideal configuration for the rotating ball MALDI interface may be orthogonal extraction [25]. In this case, the ion formation and acceleration regions are decoupled and thus the curved ball surface will have little effect on the mass resolution.

The concentrations used in this study were $100 \mu \mathrm{M}$, one to two orders of magnitude greater than desirable for common MALDI samples. Detection limit performance can be improved with better droplet deposition methods. Deposition of small droplets with high organic solvent content onto dry matrix surface may not result in good mixing and co-crystallization of matrix and analyte prior to solvent evaporation and some of the analyte may deposit on the top of the matrix. Consequently, when the sample passes under the gasket, sample loss may occur because of the mechanical force. A modified ball surface that promotes co-crystallization and limits mechanical sample loss may improve of rotating ball interface detection limit and mass range. Separate single drop deposition of both matrix and analyte on the rotating ball is another way to improve the mixing and, at the same time, minimize overall reagent consumption. The concentration detection limit can also be improved by on-target sample concentration, for example using patterned hydrophobic surfaces [2]. Decreasing the sample spots from 250 to $25 \mu \mathrm{m}$ in diameter could improve the concentration detection by a factor of 100 if the desorption laser can be focused onto the smaller target spot.

\section{Conclusions}

We have demonstrated an on-line rotating ball inlet coupled with a piezoelectric-actuated single droplet generator, which provides a means for picoliter volume deposition and femtomole sample detection with MALDI-TOF MS. The unique properties of the new rotating ball interface are the easily accessible atmospheric pressure sample deposition and vacuum detection regions. Single droplets of $100 \mathrm{pL}$ volume were deposited on the rotating ball with a placement precision of $400 \mu \mathrm{m}$. Detection of peptides and small proteins in low fmol quantities was readily accomplished. Future work is aimed at developing single droplet deposition with microfluidic devices and further improvements in detection limit and mass resolution. Implementation of on-line single droplet deposition for MALDI can be readily adapted to microfluidic devices using either piezoelectric or pneumatic ejection of droplets. For multiplexing, these droplets can be ejected into adjacent tracks on the rotating ball or cylinder. Stepper motor control of the rotation axis will improve the speed of analysis and allow the rapid and precise placement of the spots directly under the desorption laser.

\section{Acknowledgments}

This research is supported by the National Institutes of Health under grant no. R21RR15016 and the National Science Foundation under grant no. CHE-0196568. The authors thank Margaret C. Henk of the Socolofsky Microscopy Center for help in obtaining the SEM images.

\section{References}

1. Stump, M. J.; Fleming, R. C.; Gong, W. H.; Jaber, A. J.; Jones, J. J.; Surber C. W.; Wilkins, C. L. Matrix-Assisted Laser Desorption Mass Spectrometry. Appl. Spectrosc. Rev. 2002, 37, 275-303.

2. Schürenberg, M.; Luebbert, C.; Eickhoff, H.; Kalkum, M.; Lehrach, H.; Nordhoff, E. Prestructured MALDI-MS Sample Supports. Anal. Chem. 2000, 72, 3436-3442.

3. Switzer, G. L. A Versatile System for Stable Generation of Uniform Droplets. Rev. Sci. Instrum. 1991, 62, 2765-2771.

4. Little, D. P.; Cornish, T. J.; O'Donnell, M. J.; Braun, A.; Cotter, R. J.; Koster, H. MALDI on a Chip: Analysis of Arrays of Low-Femtomole to Subfemtomole Quantities of Synthetic Oligonucleotides and DNA Diagnostic Products Dispensed by a Piezoelectric Pipet. Anal. Chem. 1997, 69, 4540-4546.

5. Laurell, T.; Wallman, L.; Nilsson, J. Design and Development of a Silicon Microfabricated Flow-Through Dispenser for On-Line Picolitre Sample Handling. J. Micromech. Microeng. 1999, 9, 369-376.

6. Miliotis, T.; Kjellstrom, S.; Nilsson, J.; Laurell, T.; Edholm, L. E.; Marko-Varga, J. Ready-Made Matrix-Assisted Laser Desorption/Ionization Target Plates Coated with Thin Matrix Layer for Automated Sample Deposition in High Density Array Format. Rapid Commun. Mass Spectrom. 2002, 16, 117126.

7. Le, H. P. Progress and Trends in Ink-Jet Printing Technology. J. Imag. Sci. Tech. 1998, 42, 49-62.

8. Onnerfjord, P.; Nilsson, J.; Wallman, L.; Laurell, T.; MarkoVarga, G. Picoliter Sample Preparation in MALDI-TOF MS Using a Micromachined Silicon Flow-Through Dispenser. Anal. Chem. 1998, 70, 4755-4760.

9. Ekstrom, S.; Ericsson, D.; Onnerfjord, P.; Bengtsson, M.; Nilsson, J.; Marko-Varga, G.; Laurell, T. Signal Amplification Using Spot-on-a-Chip Technology for the Identification of Proteins via MALDI-TOF MS. Anal. Chem. 2001, 73, 214-219.

10. Allmaier, G. Picoliter to Nanoliter Deposition of Peptide and Protein Solutions for Matrix-Assisted Laser Desorption/Ionization Mass Spectrometry. Rapid Commun. Mass Spectrom. 1997, 11, 1567-1569.

11. Ericson, C.; Phung, Q. T.; Horn, D. M.; Peters, E. C.; Fitchett, J. R.; Ficarro, S. B., Salomon, A. R.; Brill, L. M.; Brock, A. An Automated Noncontact Deposition Interface for Liquid Chromatography Matrix-Assisted Laser Desorption/Ionization Mass Spectrometry. Anal. Chem. 2003, 75, 2309-2315.

12. Lee, D. S.; Aerni, H.-R.; Erskine, A. R.; Reyzer, M. L.; Cornett, D. S.; Mutz, M. W.; Caprioli, R. M.; Ellson, R. N. Automation of Sample Preparation for MALDI-TOF Mass Spectrometry. Proceedings of the 6th International Symposium on Mass Spectrometry in the Health and Life Sciences; San Francisco, CA, August, 2003.

13. Wall, D. B.; Berger, S. J.; Finch, J. W.; Cohen, S. A.; Richardson, K.; Chapman R.; Drabble, D.; Brown, J.; Gostick, D. Continuous Sample Deposition from Reversed Phase Liquid Chromatography to Tracks on a Matrix-Assisted Laser Desorption/ Ionization Precoated Target for the Analysis of Protein Digests. Electropheresis 2002, 23, 3194-3204. 
14. Zhang, B.; McDonald C.; Li, L. Combining Liquid Chromatography with MALDI Mass Spectrometry Using a Heated Droplet Interface. Anal. Chem. 2004, 76, 992-1001.

15. Whittal, R. M.; Keller, B. O.; Li, L. Nanoliter Chemistry Combined with Mass Spectrometry for Peptide Mapping of Proteins from Single Mammalian Cell Lysates. Anal. Chem. 1998, 70, 5344-5347.

16. Keller, B. O.; Li, L. Nanoliter Solvent Extraction Combined with Microspot MALDI TOF Mass Spectrometry for the Analysis of Hydrophobic Biomolecules. Anal. Chem. 2001, 73, 2929-2936.

17. Onnerfjord, P.; Ekstrom, S.; Bergqiust J.; Nilsson, J.; Laurell, T.; Marko-Varga, G. Homogeneous Sample Preparation for Automated High Throughput Analysis with Matrix-Assisted Laser Desorption/Ionisation Time-of-Flight Mass Spectrometry. Rapid Commun. Mass Spectrom. 1999, 13, 315-322.

18. Miliotis, T.; Marko-Varga, G; Nilsson, J.; Laurell, T. Development of Silicon Microstructures and Thin-Film MALDI Target Plates for Automated Proteomics Sample Identifications. J. Neurosci. Methods 2001, 109, 41-46.

19. Ekstrom, S.; Onnerfjord, P.; Nilsson, J.; Bengtsson, M.; Laurell, T.; Marko-Varga, G. Integrated Microanalytical Technology Enabling Rapid and Automated Protein Identification. Anal. Chem. 2000, 72, 286-293.
20. Miliotis, T.; Kjellstrom, S.; Nilsson, J.; Laurell, T.; Edholm L. E.; Marko-Varga G. Capillary Liquid Chromatography Interfaced to Matrix-Assisted Laser Desorption-Ionization Time-of-Flight Mass Spectrometry Using an On-Line Coupled Piezoelectric Flow-Through Microdispenser. J. Mass Spectrom. 2000, 35, 369-377.

21. Preisler, J.; Foret, F.; Karger, B. L. On-Line MALDI-TOF MS Using a Continuous Vacuum Deposition Interface. Anal. Chem. 1998, 70, 5278-5287.

22. Ørsnes, H.; Graf, T.; Degn, H.; Murray, K. K. A Rotating Ball Inlet for On-Line MALDI Mass Spectrometry. Anal. Chem. 2000, 72, 251-254.

23. Musyimi, H. K., Narcisse, D. A., Zhang, X., Soper, S. A., Murray, K. K. Online CE-MALDI-TOF-MS Using a Rotating Ball Interface. Anal. Chem., in press.

24. Lazar, I. M.; Ramsey, R. S.; Jacobson, S. C.; Foote, R. S.; Ramsey, J. M. Novel Microfabricated Device for Electrokinetically Induced Pressure Flow and Electrospray Ionization Mass Spectrometry. J. Chromatogr. A 2000, 892, 195-201.

25. Baldwin, M. A.; Medzihradszky, K. F.; Lock, C. M.; Fisher, B.; Settineri, T. A.; Burlingame, A. L. Matrix-Assisted Laser Desorption/Ionization Coupled with Quadrupole/Orthogonal Acceleration Time-of-Flight Mass Spectrometry for Protein Discovery, Identification, and Structural Analysis. Anal. Chem. 2001, 73, 1707-1720. 